DOI: $10.15393 /$ j3.art.2021.10950

UDC 517.54

A. S. Afanaseva-Grigoreva, E. G. Prilepkina

\title{
ON THE P-HARMONIC RADII OF CIRCULAR SECTORS
}

\begin{abstract}
It is proved that the property of logarithmic concavity of the conformal radius of a circular sector (considered as a function of the angle) extends to the domains of Euclidean space. In this case, the conformal radius is replaced by $p$-harmonic one, and the fundamental solution of the Laplace $p$-equation acts as logarithm. In the case of $p=2$, the presence of an asymptotic formula for the capacity of a degenerate condenser allows us to generalize this result to the case of a finite set of points. The method of the proof leads to the solution of one particular case of an open problem of A. Yu. Solynin.
\end{abstract}

Key words: condenser capacities, conformal radius, harmonic radius, family of curves

\section{Mathematical Subject Classification: $31 B 15$}

1. Introduction. The concept of the inner (conformal) radius plays an important role in the geometric theory of functions. For a simply connected planar domain $D$ of hyperbolic type, the conformal radius $R(D, a)$ at the point $a \in D$ is defined as modulus of the derivative at the zero of the conformal map of the unit disc onto $D$ that takes zero to $a$. In a more general situation, the definition of the conformal radius is given in terms of the Green function. In the proof of Theorem 2 in [4], when searching for the minimum of the discrete Green energy of an annulus, the property of logarithmic concavity of the conformal radius of the circular sector (considered as a function of the angle of a given sector) plays an important role. Namely, we denote by $R(\varphi)$ the conformal radius at the point $a$ of the annular sector $\left\{z=r e^{i \theta}: t<r<T,|\theta|<\pi \varphi\right\}, 0<\varphi \leqslant 1$, where $t, T, 0 \leqslant t<T \leqslant \infty, a, t<a<T$, are fixed. Then we have the inequality

$$
\frac{1}{n} \sum_{k=1}^{n} \log R\left(\varphi_{k}\right) \leqslant \log R\left(\frac{1}{n} \sum_{k=1}^{n} \varphi_{k}\right)
$$

(C) Petrozavodsk State University, 2021 
for $0<\varphi_{1}<\ldots<\varphi_{n} \leqslant 1$. Taking into account the continuity of the function $R(\varphi)$, the inequality (1) is equivalent to the concavity of the function $\log R(\varphi)$, or the inequality

$$
\frac{\log R(\alpha)+\log R(\beta)}{2} \leqslant \log R\left(\frac{\alpha+\beta}{2}\right),
$$

where $0<\alpha<\beta$. To prove inequality (1), the author used suitable conformal mappings, a radial averaging transformation, and some geometric considerations.

A natural extension of the concept of conformal radius from the plane to the Euclidean space $\mathbb{R}^{d}$ is the $p$-harmonic radius introduced in [10]. For $d=2$ and $p=2$, the $p$-harmonic radius coincides with the inner (conformal) radius. Specialists are most interested in the properties of the harmonic $(p=2)$ [2] and conformal $(p=d)$ radius [13]. The question about the validity of the inequality (1) arises in Euclidean space when the conformal radius is replaced by the $p$-harmonic radius. Since the only conformal mappings in Euclidean space are the Möbius mappings, the argument used to prove (1) does not apply.

In this note, we show that the analogue of (1) is also valid in Euclidean space. In the case $p=2$, the asymptotic formula for the degenerate condenser allows generalizing (1) to the case of several points. It remains an unsolved problem to obtain an asymptotic formula for the $p$-capacity of a degenerate condenser whose plates contract into a finite number of points.

The proofs of the theorems are based on the moduli technique for families of curves. The key ingredient in the proofs is Lemma 1. Note also that our approach is applicable not only to circular sectors, but also to some other domains. For example, in this way we obtained a solution to one particular case of an open problem of A. Yu. Solynin [3, Problem 4].

Functionals related to linear elliptic partial differential equations (such as capacity, energy, harmonic radius, etc.) and depending on the domain $D$ have a large number of applications in conformal mappings, potential theory and mechanics. Accordingly, information on the behavior of these functionals under various perturbations of the boundary $\partial D$ is of interest (see, e.g., [6], [9], [11], and references therein). Theorem 1 of this paper in the case of $p=2$ (but not arbitrary $p>1$ ) can be obtained from [11, Corollary 4.1], [6, p. 332] by choosing a suitable subharmonic function to describe the variation of the sector boundary. The technique we have presented also makes it possible to obtain, for $p=d$, an analogue 
of Laugesen's result on the concavity of the Green energy in a ball [9, Theorem 9].

2. Main results. Everywhere below, $\mathbb{R}^{d}$ means the $d$-dimensional Euclidean space of points $\mathbf{x}=\left(x_{1}, \ldots, x_{d}\right), d \geqslant 2$, with the usual norm $\|\cdot\|$ and distance $\left\|\mathbf{x}_{1}-\mathbf{x}_{2}\right\|$. For $p>1$, we denote by $\mu_{p}(\tau)$ the function

$$
\mu_{p}(\tau)=\left\{\begin{array}{l}
-\log (\tau), p=d, \\
\frac{1}{\sigma} \tau^{-\sigma}, \sigma=\frac{d-p}{p-1}, \tau>0, p \neq d .
\end{array}\right.
$$

Note that $\mathcal{E}_{p}(\mathbf{x})=\mu_{p}(\|\mathbf{x}\|)$ is the fundamental solution of the $p$-Laplace equation [10]

$$
\triangle_{p} u=-\operatorname{div}\left(|\nabla u|^{p-2} \nabla u\right)=d \omega_{d} \delta(\mathbf{x}),
$$

where $\omega_{d}$ is the volume of the unit ball and $\delta$ is the Dirac $\delta$-function. The function inverse to $y=\mu_{p}(\tau)$ is defined by the equality

$$
\nu_{p}(y)=\left\{\begin{array}{l}
\exp (-y), p=d \\
(\sigma y)^{-1 / \sigma}, \quad p \neq d
\end{array}\right.
$$

B. E. Levitskii in the paper [10] gave two equivalent definitions of the $p$-harmonic radius: in terms of the $p$-harmonic Green function and in terms of the $p$-capacity of a condenser. We will use his second definition. A condenser $C=(E, F)$ in the space $\mathbb{R}^{d}$ is a pair of nonempty, closed, and disjoint sets. The capacity ( $p$-capacity) of a condenser is defined as the value

$$
\operatorname{cap}_{p} C=\inf \int_{\mathbb{R}^{d}}|\nabla v|^{p} d x
$$

where the infimum is taken over all functions $v: \overline{\mathbb{R}}^{d} \rightarrow \mathbb{R}$ from $C^{\infty}\left(\mathbb{R}^{d}\right)$ vanishing in a neighborhood of $E$ and equal to 1 in a neighborhood of $F$. Let the domain $G \subset \mathbb{R}^{d}$ and $\mathbf{a} \in G$. For a sufficiently small $\tau>0$, we denote by $C(\tau, \mathbf{a}, G)$ the condenser $\left(S(\mathbf{a}, \tau), \mathbb{R}^{d} \backslash G\right)$, where $S(\mathbf{a}, \tau)=\left\{\mathbf{x} \in \mathbb{R}^{d}:\|\mathbf{x}-\mathbf{a}\|=\tau\right\}$. We define the $p$-harmonic radius of the domain $G$ at the point $\mathbf{a} \in G$ by the following identity:

$$
R_{p}(G, \mathbf{a})=\lim _{\tau \rightarrow 0+} \nu_{p}\left(\mu_{p}(\tau)-\lambda_{d}\left(\operatorname{cap}_{p} C(\tau, \mathbf{a}, G)\right)^{\frac{1}{1-p}}\right)
$$

where $\lambda_{d}=\left(d \omega_{d}\right)^{\frac{1}{p-1}}$. 
A. S. Afanaseva-Grigoreva, E. G. Prilepkina

The definition of the $p$-harmonic radius can also be formulated in terms of the moduli of families of curves [7]. Following Ahlfors [1], we will understand a curve as the union of a finite number of closed or open arcs. For a family of locally rectifiable curves $\Gamma$ and $p>1$, the module ( $p$-module) is the quantity

$$
M_{p}(\Gamma)=\inf \int_{\mathbb{R}^{d}} \rho^{p} d \mathbf{x},
$$

where the infimum is taken over all Borel functions $\rho: \mathbb{R}^{d} \rightarrow[0, \infty]$, such that $\int_{\gamma} \rho d s \geqslant 1$ for any curve $\gamma \in \Gamma$ and $d \mathbf{x}$ denotes the $d$-dimensional Lebesgue measure. The functions $\rho$ in the module definition are called admissible functions. If $G$ is some domain in $\mathbb{R}^{d}$ and all curves $\gamma \in \Gamma$ are subsets of $\bar{G}$, then the definition of a module can be limited to admissible metrics supported in $\bar{G}$. For $E \subset \bar{G}$ and $F \subset \bar{G}$, the symbol $\Gamma(E, F, G)$ will mean the family of curves connecting $E$ with $F$ in $G, M_{p}(E, F, G)$ denotes its module. Recall that the curve $\gamma(t)$ connects the sets $E$ and $F$ in the domain $G$, if $\gamma(t)$ is continuous on the interval $(a ; b), \gamma(t) \in G$ for $t \in(a ; b)$ and $\lim _{t \rightarrow a} \operatorname{dist}(\gamma(t), E)=0, \lim _{t \rightarrow b} \operatorname{dist}(\gamma(t), F)=0$.

It is known that the $p$-capacity of a condenser coincides with the $p$-module of the family of curves connecting its plates [12]. From (3) it follows that

$$
R_{p}(G, \mathbf{a})=\lim _{\tau \rightarrow 0+} \nu_{p}\left(\mu_{p}(\tau)-\lambda_{d} M_{p}(\tau, \mathbf{a}, G)^{\frac{1}{1-p}}\right),
$$

or

$$
\mu_{p}\left(R_{p}(G, \mathbf{a})\right)=-\lambda_{d} M_{p}(\tau, \mathbf{a}, G)^{\frac{1}{1-p}}+\mu_{p}(\tau)+o(1), \quad \tau \rightarrow 0 .
$$

Here $M_{p}(\tau, \mathbf{a}, G)$ is the module of the family of curves connecting the hypersphere $S(\mathbf{a}, \tau)$ of sufficiently small radius $\tau$ with the boundary $\partial G$. We obtain from (5) the asymptotic formula

$$
\begin{array}{r}
M_{p}(\tau, \mathbf{a}, G)=\left(\frac{\lambda_{d}}{\mu_{p}(\tau)}\right)^{p-1}\left(1+\mu_{p}\left(R_{p}(G, \mathbf{a})\right) \frac{(p-1)}{\mu_{p}(\tau)}+o\left(\frac{1}{\mu_{p}(\tau)}\right)\right) \\
\tau \rightarrow 0, \quad 1<p \leqslant d .
\end{array}
$$

Note that the equality of capacity and modulus and the properties of the capacity of the condenser [3, Theorem 1.10] imply

$$
M_{p}\left(\tau, \mathbf{a}, G_{1} \cup G_{2}\right)+M_{p}\left(\tau, \mathbf{a}, G_{1} \cap G_{2}\right) \leqslant M_{p}\left(\tau, \mathbf{a}, G_{1}\right)+M_{p}\left(\tau, \mathbf{a}, G_{2}\right) .
$$


The formula (6) for $1<p \leqslant d$ leads to the inequality

$$
\begin{aligned}
\mu_{p}\left(R_{p}\left(G_{1} \cup G_{2}, \mathbf{a}\right)\right)+\mu_{p}\left(R_{p}\left(G_{1} \cap G_{2}, \mathbf{a}\right) \leqslant\right. & \\
& \mu_{p}\left(R_{p}\left(G_{1}, \mathbf{a}\right)\right)+\mu_{p}\left(R_{p}\left(G_{2}, \mathbf{a}\right)\right) .
\end{aligned}
$$

Denote by $J$ the $(d-2)$-dimensional plane $\left\{\mathbf{x}=\left(0,0, x_{3}, \ldots, x_{d}\right)\right\}$. We will need the cylindrical coordinates $\left(r, \theta, \mathbf{x}^{\prime}\right)$ of the point $\mathbf{x}=\left(x_{1}, \ldots, x_{d}\right)$ in $\mathbb{R}^{d}$, related to the Cartesian coordinates by $x_{1}=r \cos \theta, x_{2}=r \sin \theta$, $\mathrm{x}^{\prime} \in J$.

For $0<\varphi \leqslant 1$, the symbol $S(\varphi)$ will denote the circular sector of angle $2 \pi \varphi$. More precisely, $S(\varphi)=S(\varphi, t, T)=\left\{\mathbf{x}=\left(r, \theta, \mathbf{x}^{\prime}\right)\right.$ : $t<\|\mathbf{x}\|<T,|\theta|<\pi \varphi\}$, where $t, T, 0 \leqslant t<T \leqslant \infty$ are fixed.

Theorem 1. Suppose that $1<p \leqslant d$, $\mathbf{a}=\left(a_{1}, 0, \ldots, 0\right) \in S(\varphi)$ and $R_{p}(\varphi)$ is the $p$-harmonic radius of the sector $S(\varphi)$ at point $\mathbf{a}$. Then the function $f(\varphi)=\mu_{p}\left(R_{p}(\varphi)\right)$ is convex on $0<\varphi \leqslant 1$.

For $p=2$, Theorem 1 can be generalized to the case of a finite set of points. To formulate our next result, we need the Green function for the Laplace operator vanishing at the points of the boundary $\partial B$ of the domain $B$. This Green function with a pole at the point $\mathbf{x}_{0} \in B$ will be denoted by $g_{B}\left(\mathbf{x}, \mathbf{x}_{0}\right)$. In the neighborhood of $\mathbf{x}_{0}$, the following expansion holds:

$$
\begin{array}{r}
g_{B}\left(\mathbf{x}, \mathbf{x}_{0}\right)=\left((d-2) s_{d-1}\right)^{-1}\left(\left\|\mathbf{x}-\mathbf{x}_{0}\right\|^{2-d}-\left(R_{2}\left(B, \mathbf{x}_{0}\right)\right)^{2-d}\right)+o(1), \\
\mathbf{x} \rightarrow \mathbf{x}_{0}, \quad d \geqslant 3, \\
g_{B}\left(\mathbf{x}, \mathbf{x}_{0}\right)=-\log \left\|\mathbf{x}-\mathbf{x}_{0}\right\|+\log \left(R_{2}\left(B, \mathbf{x}_{0}\right)\right)+o(1), \quad \mathbf{x} \rightarrow \mathbf{x}_{0}, \quad d=2,
\end{array}
$$

where $s_{d-1}=2 \pi^{d / 2} / \Gamma(d / 2)$ is the area of the unit hypersphere. Note that the definition of the Green function in different sources differs up to a multiplicative factor. Here we adhere to the definition of the Green function adopted in the [5], [3].

Theorem 2. Assume that $\mathbf{a}_{1}, \ldots, \mathbf{a}_{m} \in S(\varphi)$ belong to the axis $\left\{\mathbf{x} \in \mathbb{R}^{d}: \mathbf{x}=\left(x_{1}, 0, \ldots, 0\right)\right\}, g_{S(\varphi)}\left(\mathbf{a}_{k}, \mathbf{a}_{l}\right)$ is the Green function of the circular sector $S(\varphi)$ evaluated at the point $\mathbf{a}_{k}$ with the pole at the point $\mathbf{a}_{l}$,

$$
\left.G(\varphi)=\sum_{k=1}^{m} R_{2}\left(S(\varphi), \mathbf{a}_{k}\right)\right)^{2-d}-(d-2) s_{d-1} \sum_{k=1}^{m} \sum_{\substack{l=1 \\ l \neq k}}^{m} g_{S(\varphi)}\left(\mathbf{a}_{k}, \mathbf{a}_{l}\right), d \geqslant 3
$$




$$
\left.G(\varphi)=-\sum_{k=1}^{m} \log R_{2}\left(S(\varphi), \mathbf{a}_{k}\right)\right)-\sum_{k=1}^{m} \sum_{\substack{l=1 \\ l \neq k}}^{m} g_{S(\varphi)}\left(\mathbf{a}_{k}, \mathbf{a}_{l}\right), d=2 .
$$

Then the function $G(\varphi)$ is convex on $0<\varphi \leqslant 1$.

To prove these theorems, let us compare the moduli of some families of curves. The family $\Gamma_{2}$ is said to be longer than the family $\Gamma_{1}$ if each curve from $\gamma_{2} \in \Gamma_{2}$ has a subcurve $\gamma_{1} \in \Gamma_{1}$. In this case, $M_{p}\left(\Gamma_{2}\right) \leqslant M_{p}\left(\Gamma_{1}\right)$.

Let $L$ be some hyperplane that splits $\mathbb{R}^{d}$ into two closed half-spaces $\Lambda^{+}$and $\Lambda^{-}$. For any set $A \subset \mathbb{R}^{d}$, we denote $A^{+}=A \cap \overline{\Lambda^{+}}, A^{-}=A \cap \overline{\Lambda^{-}}$; and let $A^{*}$ be the set symmetric to $A$ with respect to $L$.

Lemma 1. Suppose that the hyperplane $L$ intersects some bounded Jordan measurable domain $G \subset \mathbb{R}^{d},\left(G^{-}\right)^{*} \subset G^{+}$, and curves from the families $\Gamma=\{\gamma\}, \Gamma_{1}=\left\{\gamma_{1}\right\}, \Gamma_{2}=\left\{\gamma_{2}\right\}$ satisfy the conditions: $\gamma \subset \overline{G^{+}}, \gamma_{1} \subset \overline{G^{+} \backslash\left(G^{-}\right)^{*}}, \gamma_{2} \subset \bar{G}$. Let $\Gamma_{0}$ denote the family of curves $\gamma_{0}=\gamma \cup\left(\gamma \cap \overline{\left(G^{-}\right)^{*}}\right)^{*}, \gamma \in \Gamma$. If $\Gamma$ is longer than $\Gamma_{1}$ and $\Gamma_{0}$ is longer than $\Gamma_{2}$, then

$$
2 M_{p}(\Gamma) \leqslant M_{p}\left(\Gamma_{1}\right)+M_{p}\left(\Gamma_{2}\right)
$$

We apply Lemma 1 to the circular sectors in the Theorem 1 . If instead of circular sectors we consider curvilinear trapezoids, we obtain a solution to the special case of the open problem [3, Problem 4]. This problem was posed by A. Yu. Solynin in the following formulation: let $f_{1}(x)>0, f_{2}(x)>0$ be continuous functions on the interval $a<x<b$, $-\infty \leqslant a<b \leqslant+\infty, f_{0}(x)=\left(f_{1}(x)+f_{2}(x)\right) / 2$, and let $r\left(B_{k}, x\right)$ be conformal radius of $B_{k}=\left\{z: a<\operatorname{Re} z<b,|\operatorname{Im} z|<f_{k}(x)\right\}, k=0,1,2$. It is conjectured that

$$
r\left(B_{0}, x\right) \geqslant \sqrt{r\left(B_{1}, x\right) r\left(B_{2}, x\right)}
$$

for all $x \in(a ; b)$. In Theorem 3 , it is proved that under the additional condition $f_{0}(x)=$ const, the inequality (9) is also valid in Euclidean space of any dimension.

Theorem 3. Assume that $p \in(1, d], \mathbf{x}=\left(x_{1}, \ldots, x_{d-1}\right) \in \mathbb{R}^{d-1}, f_{1}(\mathbf{x})>0$, $f_{2}(\mathbf{x})>0$ are continuous real-valued functions on some bounded Jordan measurable domain $D \in \mathbb{R}^{d-1}, f_{0}(\mathbf{x})=\left(f_{1}(\mathbf{x})+f_{2}(\mathbf{x})\right) / 2=c=$ const and $B_{k}=\left\{\left(x_{1}, \ldots, x_{d-1}, x_{d}\right) \in \mathbb{R}^{d}:\left(x_{1}, \ldots, x_{d-1}\right) \in D,\left|x_{d}\right|<f_{k}(\mathbf{x})\right\}$, $k=0,1,2$. Then

$$
2 \mu_{p}\left(R_{p}\left(B_{0}, \mathbf{a}\right)\right) \leqslant \mu_{p}\left(R_{p}\left(B_{1}, \mathbf{a}\right)\right)+\mu_{p}\left(R_{p}\left(B_{2}, \mathbf{a}\right)\right)
$$


for each $\mathbf{a} \in D$.

Corollary. Suppose that $1<p \leqslant d, h>0, P(h)$ is a parallelepiped in the space $\mathbb{R}^{d}, P(h)=\left\{\left(x_{1}, \ldots, x_{d}\right) \in \mathbb{R}^{d}:\left|x_{i}\right| \leqslant b_{i}, i=1, \ldots, d-1,\left|x_{d}\right|<h\right\}$, $r_{p}(h)$ is the p-harmonic radius of the parallelepiped $P(h)$ at the point $\mathbf{a}$, $\mathbf{a}=\left(a_{1}, \ldots, a_{d-1}, 0\right) \in P(h)$. Then the function $\mu_{p}\left(r_{p}(h)\right)$ is convex on $0<h<\infty$.

In conclusion, we note that a similar approach is applicable to the study of the properties of the $p$-harmonic Robin radius [3], [8]. For a set $Q$, lying in the closure of the domain $G$, we define the $p$-harmonic Robin radius as

$$
R_{p}(G, Q, \mathbf{a})=\lim _{\tau \rightarrow 0+} \nu_{p}\left(\mu_{p}(\tau)-\lambda_{d} M_{p}(\tau, \mathbf{a}, Q, G)^{\frac{1}{1-p}}\right),
$$

where $M_{p}(\tau, \mathbf{a}, Q, G)$ is the module of the family of curves connecting the hypersphere $S(\mathbf{a}, \tau)$ with the set $Q$ in the domain $G$. Repeating the proof of Theorem 1 with obvious changes, we obtain the convexity of the function $w(\varphi)=\mu_{p}\left(R_{p}(S(\varphi), Q(\varphi), \mathbf{a})\right.$. Here $\mathbf{a}=\left(a_{1}, 0, \ldots, 0\right) \in S(\varphi)$ and $Q(\varphi)$ means the part of the boundary $S(\varphi)$ lying on the hyperplanes $|\theta|=\pi \varphi$.

\section{Proofs.}

Proof of Lemma 1. Let $\rho_{1}$ and $\rho_{2}$ be admissible metrics for $\Gamma_{1}$ and $\Gamma_{2}$, respectively. If all curves of the family under consideration lie in some set, we assume that admissible functions are extended by zero outside this set. Consider the following metric defined on $\bar{G}^{+}$:

$$
\rho(\mathbf{x})=\frac{\rho_{1}(\mathbf{x})+\rho_{2}(\mathbf{x})+\rho_{2}\left(\mathbf{x}^{*}\right)}{2}
$$

where $\mathbf{x}^{*}$ denotes the point symmetric to $\mathbf{x}$ with respect to the hyperplane $L$. By the hypothesis of the lemma, for any curve $\gamma \in \Gamma$ there are subcurves $\gamma_{1} \in \Gamma_{1}$ and $\gamma_{2} \in \Gamma_{2}$, such that $\gamma_{1} \subset \gamma, \gamma_{2} \subset \gamma \cup\left(\gamma \cap \overline{\left(G^{-}\right)^{*}}\right)^{*}$. Then

$$
\begin{aligned}
2 \int_{\gamma} \rho(\mathbf{x}) d s & =\int_{\gamma} \rho_{1}(\mathbf{x}) d s+\int_{\gamma} \rho_{2}(\mathbf{x}) d s+\int_{\gamma} \rho_{2}\left(\mathbf{x}^{*}\right) d s= \\
& =\int_{\gamma} \rho_{1}(\mathbf{x}) d s+\int_{\gamma} \rho_{2}(\mathbf{x}) d s+\int_{\gamma \cap\left(G^{-}\right)^{*}} \rho_{2}\left(\mathbf{x}^{*}\right) d s=
\end{aligned}
$$




$$
\begin{aligned}
=\int_{\gamma} \rho_{1}(\mathbf{x}) d s+\int_{\gamma} \rho_{2}(\mathbf{x}) d s+ & \int_{\left(\gamma \cap\left(G^{-}\right)^{*}\right)^{*}} \rho_{2}(\mathbf{x}) d s \geqslant \\
& \geqslant \int_{\gamma_{1}} \rho_{1}(\mathbf{x}) d s+\int_{\gamma_{2}} \rho_{2}(\mathbf{x}) d s \geqslant 2,
\end{aligned}
$$

which means that $\rho(\mathbf{x})$ is admissible for $\Gamma$. It follows from the definition of the modulus and convexity of the function $f(x)=x^{p}$ for $p>1$ that

$$
\begin{aligned}
2 M_{p}(\Gamma) \leqslant 2 \int_{\overline{G^{+}}} \rho^{p}(\mathbf{x}) d \mathbf{x}= & 2 \int_{\overline{G^{+}}}\left(\frac{\rho_{1}(\mathbf{x})+\rho_{2}(\mathbf{x})+\rho_{2}\left(\mathbf{x}^{*}\right)}{2}\right)^{p} d \mathbf{x} \leqslant \\
& \leqslant \int_{\frac{G^{+}}{G^{+}}}\left(\rho_{1}(\mathbf{x})+\rho_{2}\left(\mathbf{x}^{*}\right)\right)^{p} d \mathbf{x}+\int_{\frac{G^{+}}{}} \rho_{2}(\mathbf{x})^{p} d \mathbf{x} .
\end{aligned}
$$

Note that the metric $\rho_{1}(\mathbf{x})$ is nonzero only on the set $\overline{G^{+} \backslash\left(G^{-}\right)^{*}}$, and the metric $\rho_{2}\left(\mathbf{x}^{*}\right)=0$ for $\mathbf{x} \in \overline{G^{+} \backslash\left(G^{-}\right)^{*}}$. Calculating the right-hand side (11), we get

$$
\begin{aligned}
& 2 M_{p}(\Gamma) \leqslant \frac{\int}{G^{+} \backslash\left(G^{-}\right)^{*}} \rho_{1}(\mathbf{x})^{p} d \mathbf{x}+\int_{\frac{\left.G^{-}\right)^{*}}{\left(G^{*}\right.}} \rho_{2}\left(\mathbf{x}^{*}\right)^{p} d \mathbf{x}+\underset{\frac{\int^{+}}{G^{+}}}{\rho_{2}(\mathbf{x})^{p} d \mathbf{x}=} \\
& =\int_{\mathbb{R}^{d}} \rho_{1}(\mathbf{x})^{p} d \mathbf{x}+\int_{\frac{G^{-}}{G^{+}}} \rho_{2}(\mathbf{x})^{p} d \mathbf{x}+\int_{\mathbb{R}^{d}} \rho_{2}(\mathbf{x})^{p} d \mathbf{x}=\int_{\mathbb{R}^{d}} \rho_{1}(\mathbf{x})^{p} d \mathbf{x}+\int_{2} \rho_{2}(\mathbf{x})^{p} d \mathbf{x} .
\end{aligned}
$$

Passing to the infimum with respect to admissible metrics $\rho_{1}$ and $\rho_{2}$ completes the proof.

Proof of Theorem 1. Let $D(\varphi)=\left\{\mathbf{x}=\left(r, \theta, \mathbf{x}^{\prime}\right): t<\|\mathbf{x}\|<T\right.$, $0<\theta<\pi \varphi\}$ be "the upper half" of the circular sector $S(\varphi)$. Suppose that $F(\varphi)$ is the part of $\partial D(\varphi)$ that does not contain points of the hyperplane $\theta=0, F(\varphi)=\partial D(\varphi) \cap \partial S(\varphi)$. The symbol $\Gamma(\varphi)$ will denote the modulus of the family of curves connecting the hemisphere $S(\mathbf{a}, \tau)^{+}=\left\{\mathbf{x}=\left(r, \theta, \mathbf{x}^{\prime}\right):\|\mathbf{x}-\mathbf{a}\|=\tau, 0 \leqslant \theta\right\}$ with the set $F(\varphi)$ in the domain $D(\varphi), \Gamma(\varphi)=\Gamma\left(S(\mathbf{a}, \tau)^{+}, F(\varphi), D(\varphi)\right)$.

Suppose $0<\alpha<\beta \leqslant 1$. Take $\tau>0$ so small that $\overline{D(\alpha)}$ contains the $S(\mathbf{a}, \tau)^{+}$. In Lemma 1 , we set $G=D(\beta)$; the hyperplane $L$ is the hyperplane containing $\left\{\mathbf{x}=\left(r, \theta, \mathbf{x}^{\prime}\right): \theta=(\alpha+\beta) / 2\right\}, \mathbf{a} \in \Lambda^{+}$; 
families of curves are $\Gamma_{1}=\Gamma(\alpha), \Gamma_{2}=\Gamma(\beta), \Gamma=\Gamma((\alpha+\beta) / 2)$. Because $D(\alpha) \subset D((\alpha+\beta) / 2)$, we see that the family of curves $\Gamma$ is longer than $\Gamma_{1}$. Let us show that the auxiliary family $\Gamma_{0}$ is longer than $\Gamma_{2}$. Any curve from $\Gamma$ connects $S(\mathbf{a}, \tau)^{+}$either with a point lying on the boundary of the annulus $\{t<\|\mathbf{x}\|<T\}$ or with a point of the hyperplane $\{\theta=\pi(\alpha+\beta) / 2\}$. In the first case, the curve $\gamma$ belongs to $\Gamma_{2}$ and the corresponding curve $\gamma_{0}=\gamma \cup\left(\gamma \cap \overline{\left(G^{-}\right)^{*}}\right)^{*} \in \Gamma_{0}$ has the subcurve $\gamma \in \Gamma_{2}$. In the second case, $\gamma$ contains a curve $\gamma_{3}$ connecting in the $\left.\left(G^{-}\right)^{*}=D((\alpha+\beta) / 2)\right) \backslash D(\alpha)$ a point $\mathbf{c} \in\{\theta=\pi(\alpha+\beta) / 2\}$ with a point $\mathbf{b} \in\{\theta=\pi \alpha\}$. Note that $\mathbf{b}^{*} \in\{\theta=\pi \beta\}$. So $\left(\gamma \cup \gamma_{3}^{*}\right)$ connects $S(\mathbf{a}, \tau)^{+}$with $F(\beta)$. It means that $\left(\gamma \cup \gamma_{3}^{*}\right) \in \Gamma_{2}$. Since $\left(\gamma \cup \gamma_{3}^{*}\right) \subset\left(\gamma \cup\left(\gamma \cap \overline{\left(G^{-}\right)^{*}}\right)^{*}\right)=\gamma_{0}$, the family $\Gamma_{0}$ is longer than $\Gamma_{2}$. Now, Lemma 1 implies the inequality

$$
2 M_{p}(\Gamma(\alpha+\beta) / 2) \leqslant M_{p}(\Gamma(\alpha))+M_{p}(\Gamma(\beta)) .
$$

Due to symmetry, $M_{p}(\tau, \mathbf{a}, S(\varphi))=2 M_{p}(\Gamma(\varphi))$. Therefore, we have

$$
2 M_{p}(\tau, \mathbf{a}, S(\alpha+\beta) / 2) \leqslant M_{p}(\tau, \mathbf{a}, S(\alpha))+M_{p}(\tau, \mathbf{a}, S(\beta)) .
$$

To obtain the required inequality

$$
\mu_{p}\left(R_{p}(S(\alpha+\beta) / 2, \mathbf{a})\right) \leqslant\left(\mu_{p}\left(R_{p}(S(\alpha), \mathbf{a})\right)+\mu_{p}\left(R_{p}(S(\beta), \mathbf{a})\right) / 2,\right.
$$

it remains to apply the asymptotic formula (6).

Proof of Theorem 2. Replacing $S(\mathbf{a}, \tau)^{+}$in the proof of Theorem 1 by $S\left(\mathbf{a}_{1}, \tau\right)^{+} \cup S\left(\mathbf{a}_{2}, \tau\right)^{+} \ldots \cup S\left(\mathbf{a}_{m}, \tau\right)^{+}$, we obtain the inequality

$$
2 \tilde{M}_{2}(\tau, \mathbf{a}, S(\alpha+\beta) / 2) \leqslant \tilde{M}_{2}(\tau, \mathbf{a}, S(\alpha))+\tilde{M}_{2}(\tau, \mathbf{a}, S(\beta)) .
$$

Here $\tilde{M}_{2}(\tau, \mathbf{a}, S(\varphi))$ stands for the $p$-module of the family of curves connecting the boundary $\partial S(\varphi)$ with $S\left(\mathbf{a}_{1}, \tau\right) \cup S\left(\mathbf{a}_{2}, \tau\right) \ldots \cup S\left(\mathbf{a}_{m}, \tau\right)$. Equality of the $p$-capacity of a condenser and the $p$-modulus of the families of curves connecting its plates allows us to use the asymptotic formula for 2-capacity of a condenser with degenerate plates (see [5, Proof of Theorem 1] in the case of $d \geqslant 3$ and [3] in the case of $d=2$ ). The required asymptotic has the form

$$
\begin{aligned}
& \frac{\tilde{M}_{2}(\tau, \mathbf{a}, S(\varphi))}{(d-2) s_{d-1}}=m \tau^{d-2}+\tau^{2(d-2)} G(\varphi)+o\left(\tau^{2(d-2)}\right) \\
& \\
& \tau \rightarrow 0, d \geqslant 3,
\end{aligned}
$$




$$
\begin{aligned}
& \frac{\tilde{M}_{2}(\tau, \mathbf{a}, S(\varphi))}{2 \pi}=-m(\log \tau)^{-1}+(\log \tau)^{-2} G(\varphi)+o\left((\log \tau)^{-2}\right) \\
& \tau \rightarrow 0, d=2
\end{aligned}
$$

The inequality (12) and the asymptotic formulas (13), (14) imply the convexity of the function $G(\varphi)$. The theorem is proved.

Proof of Theorem 3. We use the following notation: $R^{+}=\left\{\left(x_{1}, \ldots, x_{d-1}, x_{d}\right) \in \mathbb{R}^{d}: x_{d}>0\right\}, S^{+}(\mathbf{a}, \tau)=S(\mathbf{a}, \tau) \cap R^{+}$, $G_{k}=B_{k} \cap R^{+}, F_{k}=\partial B_{k} \cap \partial G_{k}, k=0,1,2$. In the case of $f_{1}(\mathbf{x}) \leqslant f_{2}(\mathbf{x})$ for $\mathbf{x} \in D$, we set $G=G_{2}, L=\left\{\left(x_{1}, \ldots, x_{d-1}, x_{d}\right) \in \mathbb{R}^{d}: x_{d}=c\right\}, \mathbf{a} \in \Lambda^{+}$, $\Gamma=\Gamma\left(S^{+}(\mathbf{a}, \tau), F_{0}, G_{0}\right), \Gamma_{1}=\Gamma\left(S^{+}(\mathbf{a}, \tau), F_{1}, G_{1}\right), \Gamma_{2}=\Gamma\left(S^{+}(\mathbf{a}, \tau), F_{2}, G_{2}\right)$. As in the proof of Theorem 1, we can verify that the chosen parameters satisfy the conditions of Lemma 1. From (8), symmetry considerations, and the asymptotic formula (6), we obtain the required inequality

$$
2 \mu_{p}\left(R_{p}\left(B_{0}, \mathbf{a}\right)\right) \leqslant \mu_{p}\left(R_{p}\left(B_{1}, \mathbf{a}\right)\right)+\mu_{p}\left(R_{p}\left(B_{2}, \mathbf{a}\right)\right) .
$$

The condition $f_{1}(\mathbf{x}) \leqslant f_{2}(\mathbf{x})$ can be removed by considering, for $\mathbf{x} \in D$, the functions

$$
\begin{aligned}
& g_{1}(\mathbf{x})=\min \left(f_{1}(\mathbf{x}), f_{2}(\mathbf{x})\right), \\
& g_{2}(\mathbf{x})=\max \left(f_{1}(\mathbf{x}), f_{2}(\mathbf{x})\right) .
\end{aligned}
$$

Denote $D_{k}=\left\{\left(x_{1}, \ldots, x_{d-1}, x_{d}\right) \in \mathbb{R}^{d}:\left(x_{1}, \ldots, x_{d-1}\right) \in D,\left|x_{d}\right|<g_{k}(\mathbf{x})\right\}$, $k=0,1,2$. Since $g_{1}(\mathbf{x}) \leqslant g_{2}(\mathbf{x}), D_{0}=B_{0}, D_{1}=B_{1} \cap B_{2}, D_{2}=B_{1} \cup B_{2}$, from the above and the inequality $(7)$ we obtain the following chain of relations:

$$
\begin{aligned}
2 \mu_{p}\left(R_{p}\left(B_{0}, \mathbf{a}\right)\right)=2 \mu_{p}\left(R_{p}\left(D_{0}, \mathbf{a}\right)\right) & \leqslant \mu_{p}\left(R_{p}\left(D_{1}, \mathbf{a}\right)\right)+\mu_{p}\left(R_{p}\left(D_{2}, \mathbf{a}\right)\right) \leqslant \\
& \leqslant \mu_{p}\left(R_{p}\left(B_{1}, \mathbf{a}\right)\right)+\mu_{p}\left(R_{p}\left(B_{2}, \mathbf{a}\right)\right) .
\end{aligned}
$$

The theorem is proved.

Acknowledgments. This work was supported by RFBR (project 2001-00018) and Ministry of Science and Higher Education of the Russian Federation (agreement No. 075-02-2021-1395)

The authors are grateful to the participants of the "Vladivostok Seminar on Analysis" for a number of valuable comments.

\section{References}

[1] Ahlfors L. V. Lectures on Quasiconformal Mappings. Princeton, N.J., Van Nostrand, 1966. 
[2] Bandle C., Flucher M. Harmonic radius and concentration of energy, hyperbolic radius and Liouvilles equations $\Delta U=0$ and $\Delta U=U^{\frac{n+2}{n-2}}$. SIAM Review, 1996, vol. 38 (2), pp. 191-238.

DOI: https://doi.org/10.1137/1038039

[3] Dubinin V. N. Condenser Capacities and Symmetrization in Geometric Function Theory Basel: Birkhauser/ Springer, 2014

DOI: https://doi.org/10.1007/978-3-0348-0843-9

[4] Dubinin V. N. Green energy and extremal decompositions. Probl. Anal. Issues Anal., 2019, vol. 8 (26), no. 3, pp. 38-44.

DOI: https://doi.org/10.15393/j3.art.2019.6730

[5] Dubinin V. N., Prilepkina E. G. On extremal decomposition of n-space domains. J. Math. Sci., 2001, vol. 105, no. 4, pp. 2180-2189.

DOI: https://doi.org/10.1023/A:1011329108587

[6] Garabedian P.R., Schiffer M. Convexity of domain functionals. J. Anal. Math., 1953, vol 2, pp. 281-368.

DOI: https://doi.org/10.1007/BF02825640

[7] Kalmykov S., Prilepkina E. Extremal decomposition problems for $p$ harmonic radius. Anal. Math., 2017, vol. 43, no. 1, pp. 49-65.

DOI: https://doi.org/10.1007/s10476-017-0103-y

[8] Kalmykov S. I., Prilepkina E. G. On the p-harmonic Robin radius in the Euclidean space. J. Math. Sci., 2017, vol. 225, no. 6, pp. 969-979.

DOI: https://doi.org/10.1007/s10958-017-3508-z

[9] Laugesen R. Extremal problems involving logarithmic and Green capacity. Duke Math. J., 1993, vol. 70, no. 2, pp. 445-480.

DOI: https://doi.org/10.1215/S0012-7094-93-07009-3

[10] Levitskii B. E. Reduced p-modulus and the interior p-harmonic radius. Dokl. Akad. Nauk SSSR, 1991, vol. 316, no. 4, pp. 812-815 (in Russian); translation in: Soviet Math. Dokl., 1991, vol. 43, no. 1, pp. 189-192.

[11] Pouliasis S. Concavity of condenser energy under boundary variations. J. Geom. Anal., 2021, vol. 31 , no. 8, pp. $7726-7740$.

DOI: https://doi.org/10.1007/s12220-020-00547-3

[12] Shlyk V. A. The equality between p-capacity and p-modulus. Sib. Math. J., 1993, vol. 34, no. 6, pp. $1196-1200$.

DOI: https://doi.org/10.1007/BF00973485

[13] Wang W. N-Capacity, $N$-harmonic radius and $N$-harmonic transplantation. J. Math. Anal. Appl., 2007, vol. 327, no. 1, pp. 155-174.

DOI: https://doi.org/10.1016/j.jmaa.2006.04.017 
Received June 19, 2021.

In revised form, October 22, 2021.

Accepted October 27, 2021.

Published online November 2, 2021.

Afanaseva-Grigoreva A. S. ${ }^{a}$

E-mail: a.s.afanasevagrigoreva@yandex.ru

Prilepkina E. G. ${ }^{a, b}$

E-mail: prilepkina.eg@dvfu.ru,pril-elena@yandex.ru

${ }^{a}$ Far Eastern Federal University, Far Eastern Center for Research and Education in Mathematics

10 Ajax Bay, Russky Island, Vladivostok 690922, Russia

${ }^{b}$ Institute of Applied Mathematics, FEBRAS

7 Radio Street, Vladivostok 690041, Russia 